\title{
On the Identical Simulation of the Entire Universe
}

\begin{abstract}
Mesut KAVAK*
A time ago, I published an article [1] about deceleration of the universe. It was especially based on uncertainty, and it explains how does matter work. In this work, it was performed some analysis of the some specific subjects as an approach such as deceleration, uncertainty, possible particle formation, black hole, gravitation, energy, mass and light speed as the elements for identical simulation computations of the entire universe as the most sensitive as possible being related that article. There are some information about escaping from black holes, event horizon lengths, viscosity of free space, re-derivation of Planck constants [2] and infrastructure of some basic laws of existence mathematically as matter is directly dependent of geometric rules. Also, some elements were given for the readers to solve some required constants as the most sensitive manner. As the constants are not enough in the name of engineering, also finally I found a working algorithm out which reduces process number of the power series to process number of the quadratic equations like calculating a root of an integer as an irrational number by solving equation; so also it can be used to calculate trigonometric values in the best manner for simulations of the entire universe besides physical constants as irrational values.
\end{abstract}

\section{Introduction}

If you want to make a simulation for the entire universe, it means you are in the worst situation; because for example if you must to use $\pi$ with a number like $10^{150}$ as a multiplier, you must know at least nearly $10^{200}$ digits of $\pi$ to calculate it for the physical threshold constants which are actually belong to Max Planck. Otherwise, you will be skipped some mass magnitudes which can be create new universes. This is not a acceptable thing in the name of engineering. Also the work does not finish with only $\pi$. The Newton's gravitational constant and light speed must be known for the same sensitivity beside actual working principle must be known. Maybe is not true; but I found out Planck constant as an irrational number [1] as required by uncertainty; but as it is dependent of light speed, also it is assumed that particles draw a perfect circle that is not possible. If we can find out Planck Length or Planck Time and light speed as irrational numbers, then for example we can easily calculate the Newton's gravitational constant in the most sensitive manner without experimental constants that such these experimental constants do not work in such a big simulation if you assume that the simulation is possible.

Universe has been emerging dependent manner of some main physical laws and mathematical rules which are actually natural results for each other. There is nothing inconceivable or unreasonable. Even if there are many laws that universe emerges over them, the most basic law and condition is seen as uncertainty of Werner Heisenberg [3] as matter owes its availability and process-ability to uncertainty as it has space, formation frequency and formation time differences [1]. It seems that the deceleration from infinite by a forcing and motion closes a curve as a virtual part of infinite brings energy out at the same ratio by the work done of the forcing work, and because of this simple formation type of matter, as there is no alternative matter sits on some the most basic geometric rules; thus this formation of universe easily will be imitable by computers as there is no difference between real life and a simulation as actually both of them are an illusion because of uncertainty.

During the analysis of the formation process, it can be easily seen that matter's any value is completely irrational as matter is not absolute because of uncertainty as uncertainty increases or decreases between absoluteness and absolute absence but its loss. It always be formed by infinite number of small energies, time differences and side effects on itself. It directly brings irrational values and infinite series out; so if you want to make an identical instant simulation for the entire universe with its future and past, it means you are in the worst situation because of the digits after the comma if you do not want to skip some energy magnitudes that for example maybe these magnitudes can create new universes. Right this point, it is a little relaxing, that even if we could not calculate a identical universe from the basic geometric and mathematical rules except experimental constants, we can use the experimental constants by comparing with the current universal placement which we have by indirect calculations and using induction and deduction methods. By this way, if we achieve the work, even we can learn actual values of the experimental constants which are actually cannot be calculated by experiments since uncertainty is not going to allow this after a point.

\section{Deceleration}

As it was said [1], [4], a matter does work even if it does work relatively to us in outer space observational area by interacting another space elements by using its own energy without external energy source or when made it worked by man-made forcing with external energy, and work can only be done as much as energy existence as kinetic energy is equal to work done. Energy is not independent of velocity, mass, distance and time; thus for creation of a mass, a motion and thus time, distance and velocity must occur without any priority to 
each other as mass is only possible with existence of energy, and energy is not independent of velocity.

As a motion occurs together with a work by the rule of Figure 1 for $y=x$ and $y=x^{2}$ functions, a mass cannot get the same mass magnitude at the smaller periods of time as the energy expended for a work which is done to create the mass closes to infinite. Work done is equal to kinetic energy; thus closing to infinite means that even the smallest mass part of universe gains a potential which is able to do infinite work; thus a mass always must get smaller mass magnitude at the smaller periods of time. This means, that work cannot be done untimely manner; thus mass cannot be formed without time, and it cannot be absolute. This is uncertainty as matter is not absolute. It has a emerging frequency. A creation motion always repeats itself, and matter appears in an interval as an illusion.

As matter is not absolute, also there is no absolute threshold value. Any value can get smaller forever, and so there will always emerge infinite time differences for an assumed infinite small mass and energy part of matter. During this appearance between $[0,1]$ second time interval, as there is no absolute threshold value, also if a mass passes a point on space, its mass effect on just-passed place will never be zero; so there will always be infinite side effects on itself even for just 1 particle which has no other sub particles, or even for the biggest masses have any type atom or particle. Because of this reason, if a particle emerges by a circular motion or a motion which closes a curve of an assumed infinite small mass part as a reference threshold value, it means that there will always be an extra sensed mass. Also again because of this reason, any mass has to be counted as distributed load like snow on ground, and Force $=$ Pressure/Area formula is always in game for any size and magnitude mass. If you use a press machine to compress a material, the material will not be compressed for the same force at a point; because it already will be separated on surface, and so pressure/area ratio will be decreased. You can hole on it by a tip for the same force as actually holing is the same with compressing but you cannot compress it more.

Because of uncertainty, there will always emerge different conditions for different sizes. For example even for the same material, electrical conductivity is different for 1 electron, 1 atom, $1 \mathrm{~kg}$ or 1 ton or in heart of stars as a side effect on itself or by external reasons even during emerging duration along 1 second from zero point. For this reason, there is always a probability that we may skip some special effects or behavior. For example we can calculate electron's orbital energy by an experiment if you create a potential difference; but you may not know electron turns around atom like planets or winds a circle or helix on its orbital and also winds this circle own atom on the same orbital at the same time. You always see a cloud as also it is very fast; so you can assume that electron turns like planets. There will no difference for mathematical calculations; but you may be covered some realities also because of some experimental constants like charge de- rived by Coulomb. Math is like a rubber. You can easily make some transformations. Certainly it is not an absolute unknown thing; but maybe we may be wrong.

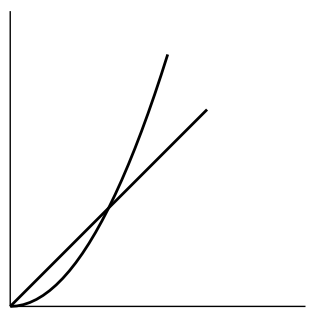

Fig. 1: Deceleration

As matter is not absolute, constant speed movement can only be created as it cannot be absolute and is relative [1], [4]. Energy can only exist in 3 main conditions that they are absolute energy, the energy which is generated by an acceleration from zero point in the other name from nothingness, and the energy caused by an acceleration which is because of a deceleration from any magnitude existent energy the infinite energy is included as well. As matter has no infinite work ability, matter cannot emerge by the acceleration from zero point because of the previous energy need.

As the formation of matter by these rules, as it was said [1] distance taken is dependent of the classic mechanics equations that they are velocity and acceleration equations; thus if it is assumed that $d$ is the distance taken, then they are dependent of $d=v t$ and $d=a t^{2}$ equation which can be expressed by the other expressions as $y=x$ and $y=x^{2}$ functions according to time as the same as on Figure 1. As the intersection point of the functions is $x=1$ second, velocity and acceleration increase to the same value at the end of 1 second. Action being the same, the road proceeded during the action performed for the matter which starts to occur after the moment of $t=0 \mathrm{sec}-$ ond must be the same road; thus this to be at the same road in $1 \geq t \geq 0$ time interval condition means that the acceleration decreases from an infinite value as a part of infinite. Deceleration cannot emerge from nothingness because of the previous energy need, and matter can only be created by forcing every second as matter is a virtual part of the infinite as you can neither detract anything from, nor add anything to infinite, since everything an element of the infinite; so it can be said that matter is absolute inert.

As work done is equal to kinetic energy and as matter is created by deceleration, a stopper opposite work on the infinite brings matter total energy out at the amount of this opposite forcing. Also to be one of them before or after of the same two opposite $F t$ works in space as $+F t$ and $-F t$ changes the displacement even momentum and energy will be conserved at that time; so if matter is formed by a circular motion there must be always a potential difference or initial movement for the resultants on Figure 2 to emerge of motion.

Any way, according to the above stated information, we must leave one of the resultants like on Figure 2 to emerge of 


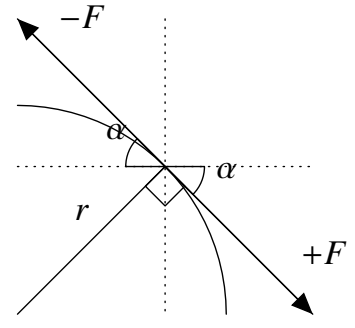

Fig. 2: The resultants

matter to calculate it. It will be enough for the calculation. Being $m v r / r=\tan (\alpha)$, for $m v r / r=\int \tan (\alpha)$ equation it will be as (1) for 1 second.

$$
\cos ^{-1}\left(e^{-m v}\right)=\alpha
$$

As required by uncertainty, mass decreases at the same ratio with time [1] for the smaller slices of time.

Actually, as matter emerges over time, for smaller application time interval than absolute upper time limit which matter gains its maximum mass magnitude value, the mass which the force is applied is smaller than general mass magnitude as the force is smaller as well; so actual equation for this condition is $F t^{2}=m t v$ where $t<t_{u}$ being $t_{u}$ upper limit which means creation time that we accept it as 1 second. Additionally, there is one more option that as motion is deterministic, when you wish to use more energy by the same force by using the force in a smaller time, there will no magnitude difference; thus $F t^{2}=m t v$ becomes invalid. This requires relative time. Time passes different for different motions. Even so, the energy is still used from total energy of universe. If this is the situation, then there are infinite times that is more suitable with uncertainty and the time differences [4], [1].

\section{Warning}

For $\frac{2 \pi r \alpha}{360}=v t$ and $\cos ^{-1}\left(e^{-m v t}\right)=\alpha$ equations, (1) turns into (2) as the below,

$$
\cos ^{-1}\left(e^{-\frac{\pi r \alpha m}{180}}\right)=\alpha
$$

and it can only be solved by solving equation as an irrational value, as actually this irrationality is required by uncertainty as well.

This angle on (2) is the angle emerges in the smallest time as required by uncertainty as a threshold value. For this angle, the smallest time in the other name the Planck Time can be found out over $\frac{2 \pi r \alpha}{360}=v t$ and the smallest distance can be found out over $\frac{2 \pi r \alpha}{360}$ as the Planck Length. These ones must be the required Planck constants for example over $m=4 \pi / c^{6}$, $v=c$ and $r=c / 2 \pi$ as they were derived [1] that is you assume that a creator wave always draws a circle that is impossible [4]. Also when the Planck length is found out over these methods, then the gravitational constant $G$ or the light speed $c$ can be found out on $G=L^{2} c^{7} / 4 \pi$ [1] being $\mathrm{L}$ is the Planck length if also one of them is known as irrational number. Otherwise, we must find out at least one of them as irrational number independent of each other manner; but the Planck time over (2) is not the same with classic one as this is a reference for sensitive calculations. It is about a frequency slip, and explained it the below.

Actually, I found out a different gravitational attraction and repulsion calculation over conservation of momentum of the waves [4] without experimental constant like $\mathrm{G}$.

\section{Warning}

If this is the situation then it can be said that Newton's gravitational constant $G$ can be calculated mathematically; but light speed must be known in the same manner as it was said the above. As light speed, Maybe it is a deceleration constant. It may completely mathematical. As universe cannot be absolute even for its any big size, light speed in the other name creation motion speed as required by entanglement must be limited and at least fixed even constant speed movement is not possible as stated the above. Because of the infinite small time differences, the same event is repeated and slowing speed is recovered; so it seems as constant and fixed speed; but as its velocity magnitude, I think it does not increase or decrease. As the first guess about this fixed light speed condition, matter's creation motion and outer space motion emerge and are accepted together [1], [4]. When it is said that an object moved then it means the circular creation motion moved by using the same space because of the deceleration from the infinite; so you can only say that the object decelerated from A to B if the object moved from A to B. This deceleration constant light speed is as a magnitude of the displacement according to the displacement time, it may always fixed as well as fixed magnitude for any size universe beyond to be fixed because of always limited universe.

Actually, I found out a some methods on possible emergence geometry of particles over conservation of momentum and energy [4]; so actually it is more suitable that we must say a motion closes curve instead of circular motion. A potential difference could not emerge with perfect circular motion.

\section{Warning}

As the second guess, matter gains its energy by only a circular motion and if the radius of the motion decreases, then as frequency increased also energy increases for the fixed speed. For the total of universe, when the radius gets smaller during a big bang, then radius of the universe which emerges after the big bang will be bigger because of the bigger centrifugal 
force and so the energy. Already I derived Planck Constant as $4 \pi / c^{4} \mathrm{Js}$ and so if you assume that light speed increased then energy will decrease as a paradox for the first look. This is because of the circular motion frequency. When the frequency increases, then detected mass or energy in an unite of volume increases. Additionally, any time is now and as required by deceleration there can only be one absolute as absolute cannot be created afterwards because of a worker before instead of the created ones; so there cannot be infinite number of universes by a constant velocity even if this velocity is not light speed we know. This is the main reason of why the Planck time which was derived over (2) is not the same with classic Planck time; because even the light speed is fixed, God can force photon to smaller radius by the same speed by using more space volume instead of using little space volume by bigger speed, and then as energy is going to increase also frequency will increase for the same speed and photon energy. At this point, Gravitational constant is the reference to learn how many slip emerged. God created by the rule of (2) or not? This is not an obstacle against finding new planets by calculating universe.

Additionally, maybe light speed can be found out over relative energy transformation [1], [4]. As I said, the upper limit is for the increasing mass is the total mass of the universe; so if we make an equation like $\frac{m_{p h}}{1-\frac{v^{2}}{c^{2}}}=m_{u}$ being $m_{p h}=4 \pi / c^{6}$ mass is the photon mass and $m_{u}=c^{12} / 2 G^{2}$ mass is the total mass of the universe, then this velocity of $v$ must be the light speed of $c$. Maybe we can make some corrections even by some series mathematically also by using (7.1) for $G$ and (2) for $L$ and so light speed.

As the deceleration, as it was said the above to emerge of a motion a potential difference is required for the two opposite resultants as matter can only be created by a circular motion. As matter has infinite number of side effect on itself because of infinite number of recovering for speed and so mass and energy as required by uncertainty, for example as any mass increasing increases the drawing mass from the space around it, also this decrease is going to effect the second condition in the other name formed mass's external effect rotation as a side effect by a deceleration which cannot recovered without an external energy. This second external deceleration is different than the creation as space and so matter always want to be fixed from the condition of to be forced creation. Because of this forcing creation condition, any matter wants to stop; but as the forcing is not removed, maximum stopping amount is seen in outer space motion in the other name usual observational space movements as an observational stop. It can be said that outer space moving objects lose their speeds if they rotate around themselves different than the friction with the space induced relative infinite small deceleration. Also this deceleration may cause the famous Bohr Jump in the other name the quantum jump.

As Gravitational constant, I think it is about geometric relation of the light speed. Let us analysis the condition under Viscosity of Free Space title by a new section.

\section{Viscosity of Free Space}

I could not realize exact calculation for the viscosity of free space yet; but I can say, that as required by Newton's third law of motion, physical magnitudes of a space wave is dependent of the density of the space which the wave emerges over it in the point of having different energy magnitudes. Also as the wave has no external energy as it uses for its movement its own total energy, the third law of motion causes energy loss in space for any mass as actually any point of the space is a gravitational field as required by entanglement because of the deceleration from the infinite as there is going to be always different densities, different than assumed zero resistance of the space. There is never going to be an emptiness even if space will be assumed as zero resistance as required by uncertainty as a reference. Also by the way, this condition may cause high pressure area in space by disrupting the vacuum at the total energy amount as particles emerge over the space which is counted as vacuum area; because if you think that there is no other particle in the universe, then if a particle emerges, it stretches the space and the universe will get smaller; so if you create a vacuum area in a tube has no air on the earth, maybe then the temperature does not be like in space because of this.

As required by Newton's third law of motion, as photon is created over space [1] as a potential difference and wave which has its own energy, it can be said that if free space was denser, photon would be heavier than the previous condition for the same energy. It means that at this condition speed decreases and mass increases for the same energy. If photon was lighter in the same space, then speed would increase instead of it. In the other hand, actually photon's energy is $4 \pi / c^{4}$ Js and its mass is $4 \pi / c^{6} \mathrm{Kgs}$ as irrational values as required by uncertainty; so if speed decreases, then it means mass increases by the square of the decrease. This may be the reason of the relation between distance and attraction force; because gravity is the same with formation of a photon. As gravity is photon induced friction in the stretched space as matter can only be formed by photon and a circular motion [1] this is the same event with a single photon's formation in space.

Actually I discovered, that the actual reason of gravitation is density differences. Denser space is more disordered because you need more speed to create denser area. If you create this denser area over a fixed constant speed, then it is still valid; but even you do not need more speed than the fixed speed, you will converge total energy of the universe for the same speed and after that you will need more speed. During this process, for the creating denser area with a fixed speed, only disorder will increase as long as you create more and more denser area. This is incompressibility of matter. If you create a lower density area, it wants to be fixed by an opposite low force; but if you create denser area, the opposite force will be bigger. Gravity works over this principle. Grav- 
itational waves frequency is the same an nearly $v=\frac{1}{t_{0}}$ where $t_{0}=\frac{3 \zeta(3)}{4 \pi c^{3}}$. Each wave creates a potential difference and when they intersected the space moves according to the condition. As matter emerge over this sliding space, also matter moves. [4]

\section{Warning}

Actually speed cannot decrease and it is for an extraction; but if it was real, the energy of a photon would decrease. This is a paradox for $4 \pi / c^{4} \mathrm{Js}$ energy in the point of decreasing energy which is caused by increasing speed; because energy is directly proportional with speed. Then this means that helical circular frequency [1] increases and detected mass in an unit of volume is preserved. It means that the ratio or the multiplication of frequency $(c / 2 \pi r)$ and energy $\left(4 \pi / c^{4}\right)$ or frequency $(c / 2 \pi r)$ and mass $\left(4 \pi / c^{6}\right)$ is fixed constantly as a constant. Now the question is this that by the defined element, how can we extract the gravitational interaction being different than the Newton's?

In the same manner as the second guess, they can be given as the other elements for the gravitational constant that as matter actually draws 3D even if it moves in 2D [1] because of the circular motion realized around itself of $2 \mathrm{D}$, the mass density can be assumed as in 2D that maybe charge is a mutual matching condition even for any small movement during the rotation of 2D around itself for two particles, and maybe it can cause a cold fusion; so for $m_{1} / \pi r^{2}$ mass which is the mass at the distance of $r$, the other mass which is emerging on this stretched space will be $m_{1} m_{2} / \pi r^{2}$ as required by Newton's third law of motion as the space is going to be denser because of the other mass. If this is the condition, then because of this $2 \mathrm{D}$ total mass against 3D total mass, this multiplication of $m_{1} m_{2} / \pi r^{2}$ must decrease as the masses is going to get smaller mass magnitudes relatively to each other, and this calculation is going to only be for linear and static condition.

Also mass is not only placed at a point even as distributed mass because of the circular motion of matter's creation photon; because as mass density is on the surface [1], it will move matter forth and back and even if the same two opposite works like $+F t$ and at the end of the $t$ time $-F t$ are applied for an object in space, it will change the displacement.

By the way as the third law of motion again, there is going to emerge a subject like vibration stopper densities. Let us analysis the condition in a new section.

\section{Vibration Stopper Densities}

As masses lose their energies in a gravitational field and any point of the space is a gravitational field because of the entanglement caused by the deceleration from the infinite as there is not going to be absolute threshold value because of solute matter as gravity effects from infinite distance at the light speed, then space can be assumed as a vibration stopper or reducer density since matter is formed at a frequency. The most extreme densities of the universe are black holes. There is a limit to be a black hole and after this limit, if you do not ask the black hole's life time, any size of black hole can emerge for any radius. In a black hole being $h$ is the Planck constant, $v$ is the frequency of the photon, $m_{b}$ is the black hole's mass, $r_{b}$ is the black hole's planetary radius, for (3) the below

$$
\int_{0}^{x} \frac{\left(h v / c^{2}\right) m_{b} G}{\left(x+r_{b}\right)^{2}} d_{x}=h v
$$

the even horizon's length will be as (4) the below.

$$
x=\frac{r_{b}^{2} c^{2}}{m G-r_{b} c^{2}}
$$

As Schwarzschild radius, on (3) when $x=0$ meter the Schwarzschild radius will be as (5) the below with zero event horizon length.

$$
r_{b}=\frac{m_{b} G}{c^{2}}
$$

and also it verifies the kinetic energy correction that as it was said [1] during motions, because of the creation at the fixed light speed, it can be said that light speed is the highest speed, and the lower is not possible as well. The condition does not allow kinetic energy to be like $w=\int_{0}^{v} v d_{m v}$; because the change amount of a mass at low speeds can be assumed as zero, and the speed always must be fixed and the light speed; thus these two conditions do not allow for integration separately even if actually they are the natural results for each other.

According to the work done by gravity, for kinetic energy correction as fixed light speed does not allow for the integration of kinetic energy

$$
\frac{m_{o} m_{b} G}{r_{b}^{2}} \cdot r_{b}=m_{o} c^{2}
$$

the Schwarzschild radius shall be as (6) the above being $m_{o}$ is the object's mass, $m_{b}$ is the black hole's mass, $r_{b}$ is the black hole's planetary radius. As mass has $K g s$ unit, for any time between $[0-1]$ second, the work done and the kinetic energy have the same decrease of magnitude in the smaller periods of time; thus for the fixed speed of light and the fixed radius, this surface has the radius of (5) destroys every small string emerging each Planck time because of the space density or tension by reducing and stopping its vibration.

In the same manner, the other black hole [1] has the radius of (7) as the below

$$
r=\sqrt{\frac{m G}{c}}
$$

where the gravitational acceleration is equal to the magnitude of the light speed of $c$, the photon loses its energy at the end of 1 second. By the way, I noticed that the Planck Length which I derived [1] as (7.1) the below

$$
L=\sqrt{\frac{4 \pi G}{c^{7}}}
$$


is completely the radius of a black hole as (7) created by a photon has 1 frequency. Also as it was said [1], If the extra radiation from outside was focused on a particle as a laser beam, since matter can perform action until the extent of its total energy and since vibrations caused by temperature increase will be deemed motion, it would turn matter into photon by vibrating at the emergence frequency about gamma frequency according to the relativistic energy transfer that there is an uncertainty for the speed limit which renders possible $v=c$ on the energy transformation; so the black holes which have this radius (7) may cause gamma ray burst by some periods when the central mass temperature causes a vibration at creation frequency, and the visible light with the gamma rays may be because of decreasing gamma rays caused by the black hole's own dense body. This burst may render visible the black hole as the black hole lose mass and so gravity. Such a dense mass may not let fusion because of mass repel. Photon cannot turn to create particle on dense space after a great density; so you can only see a temperature increase as also the vibration caused by temperature has a shock effect against gravity or electrical charge. If you ask why do the rays travel at two opposite ways, it may occur because of the helical motion.

When (5) is used on (4), then the event horizon becomes infinite; but if (7) is used on (4), then an event horizon occurs and it will be as (8) the below, and it starts from the planetary surface.

$$
x=\frac{m c G}{m G-\sqrt{m c^{3} G}}
$$

Also because of $m v^{2} / r$ force which is gotten by orbital objects light is included as well, escaping is possible even there will be a deformation amount in the escape time; because an uncertainty of the force line condition will emerge as gravity is calculated from the center of objects even for the planetary surface of black holes. For (9.1) the below

$$
\frac{m_{o} v^{2}}{r+x} \geq \frac{m_{o} m_{b} G}{(r+x)^{2}}
$$

being $r$ is (7) and $x$ is (4), the equation must always be provided at least for $v=c$ as (9) the below

$$
x \geq \frac{m G}{c^{2}}-\sqrt{\frac{m G}{c}}
$$

and this is an absolute mass repulsion even for light as a hyperbolic perfect mirror. For different $\mathrm{x}$ values which are not related with event horizon between $[0, \mathrm{x}]$, the repulsion can be seen within different times.

By the way, there can be another event similar with this and the deceleration that it can be seen at the outer universe in the other name non-observational universe. As the universe has been emerging by a circular motion and is not absolute, during the photon's emergence over this space, photon which moves linear manner will jump, slide at some points with some periods constantly because of the initial movement as the displacement is going to change; so light cannot come here after a distance. Nothing is perfect because of uncertainty. Also this can be easily the reason of the pioneer anomaly. As space stuff cannot protect their speeds because of the friction with space as a soft collision, also it will turn according to rotation direction of the universe as it emerged by a centrifugal force.

As the Schwarzschild radius destroys the photon's energy for 1 second [1] without event horizon, if $r_{b}=m_{p} G / c^{2}$ Schwarzschild radius is put on its place on (10.1) the below

$$
F_{2}=\frac{m_{o} m_{p} G}{r^{2}}
$$

then it will be as (10).

$$
F_{1}=\frac{m_{0} c^{4}}{m_{p} G}
$$

This force destroys the energy of the photon in 1 second as it increases the density of the space. If (10) destroys in 1 second, then another interaction for the same planet mass and object with a different radius as $F_{2}=m_{o} m_{p} G / r^{2}$ destroys in $F_{1} / F_{2}$ second as required by Newton's the third law of motion as (11).

$$
t_{p}=\frac{r^{2} c^{4}}{m_{p}^{2} G^{2}}
$$

The same is acceptable for atomic particles and thus all heavenly bodies, and as any point of space is a gravitational field as required by entanglement because of the deceleration from the infinite, any object loses its energy in gravitational field. Even if there is no other mass in universe, mass loses its energy because of the friction with space being it is a side effect on itself even if the resistance of the space is assumed as zero since it is not actually zero.

As the visible light loss, a visible light loses its energy in gravitational field actually the other photons are included as well, and a dim occurs. If the same energy visible lights suddenly emerge near a planet at the same time, the closer one's loss amount will be more than the further emerging one for the same time; so even if we cannot know all the moves of the universe to determine a certain visible light event horizon, if we can say it started from that place for the visible light, for (12.1) the below

$$
\int_{0}^{c t} \frac{h\left(v_{1}-v_{2}\right)(r-x)^{2} c^{4}}{h v_{1} m_{p}^{2} G^{2}} d_{r}=t
$$

it will be (12) as

$$
t=\frac{\left(v_{1}-v_{2}\right)\left(r^{3}-(r-c t)^{3}\right) c^{4}}{3 v_{1} m_{p}^{2} G^{2}}
$$

by solving equation, and it is a fall from $v_{1}$ frequency to $v_{2}$, and the visible light event horizon will be (13) as the below

$$
x=r-c t
$$


being $\mathrm{c}$ is the light speed and $\mathrm{r}$ is the distance from the center of the planet. The same is acceptable for atomic particles and so all heavenly bodies. You can observe some heavenly bodies like black hole even if the object is not a black hole.

Also it can be said that high energy cosmic particles which escaped from a black hole can easily lose their forms and become a different energy particle since gravity sucks energy by space frictions and oneself frictions by superposition of increasing masses. The inner vacuum [1] area can lose its form like a car tire and maybe it cannot be recovered. Mass spectrometers can be used for the determination of the condition by using the high energy cosmic particles. It may be healer or damager; because for example $\mathrm{D}_{2} \mathrm{O}$ is also water known as heavy water but it is toxic. We should collect high energy particles or the other particles which are created by a big size star or smaller than the earth planet in the space, that they have different energy and vibration.

\subsection{The Arc Side}

It is possible to compute trigonometric functions and $\pi$ like calculating a root of an integer. It can reduce process number of power series.

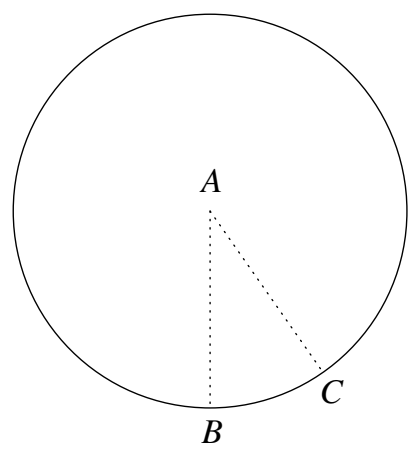

Fig. 3: Two points on the circumference

As the first step, let choosing two points like $B$ and $C$ on Fig. 3, and know that; $\angle B A C$, the radius and so $\overparen{B C^{\prime}}$ are known. The angle should not be more than $45^{\circ}$. The other angles between $45^{\circ}-90^{\circ}$ will be found out by using half angle formula of $\cos (2 \alpha)=\cos ^{2}(\alpha)-\sin ^{2}(\alpha)$. Otherwise its projection must be drawn for the other side of $A B$.

Thereupon as the second step, open $\overparen{B C}$ as $B C^{\prime}$ where the radius is the height as the same as on Fig. 4, and lengthen it as $2|\overparen{B C}|$. Know that, as $|\overparen{B C}|$ is known, also $\left|B C^{\prime}\right|$ and so $\left|A C^{\prime}\right|$ are known.

If a circle is drawn for $A C^{\prime}$ radius, then it will look like on Fig. 5, and know that $C$ point is not on $E G$. The equation becomes Eq. (14),

$$
|A D|=\frac{|A E|^{3}}{|A F|^{2}}
$$

where $|A G|=|A E|^{2} /|A F|$ in $\triangle A E F$ and $|A D|=|A G|^{2} /|A E|$ in $\triangle A G E$.

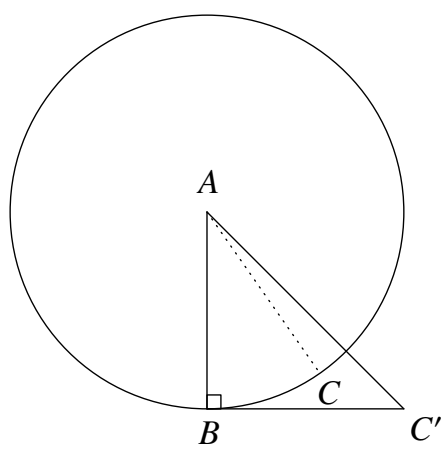

Fig. 4: The arc side

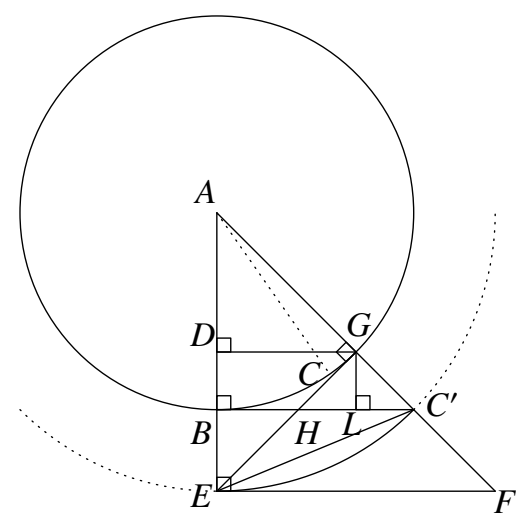

Fig. 5: The second radius

It will be as Eq. (14a),

$$
|D G|=\frac{|A B| \cdot\left|B C^{\prime}\right|}{\sqrt{|A B|^{2}+\left|B C^{\prime}\right|^{2}}}
$$

where $|A D| /|A B|=|D G| /\left|B C^{\prime}\right|$ from the similarity in $\triangle A B C^{\prime}$, $|A D|=|A G|^{2} /|A E|$ by using Euclid relation in $\triangle A G E$ and $|A E|=\sqrt{|A B|^{2}+\left|B C^{\prime}\right|^{2}}$ equation for $|A B|=|A G|$ and $|E G|=$ $\left|B C^{\prime}\right|$.

For Eq. (14), $|D B|=|A B|-|A D|$ equation turns into Eq. (14b).

$$
|D B|=|A B|-\frac{|A E|^{3}}{|A F|^{2}}
$$

As the equations are $|D B|=|G L|,|G H|=|B H|$ and $|E H|=$ $\left|H C^{\prime}\right|$, for $|G L| /|B E|=|G H| /|H E|$ it will be as $|D B| /(|A E|-$ $|A F|)=|G H| /\left(\left|B C^{\prime}\right|-|G H|\right)$, and when it is edited, it turns into Eq. (14c).

$$
|G H|=\frac{\left|B C^{\prime}\right| \cdot|D B|}{|A E|-|A D|}
$$

As $\triangle E B H$ and $\triangle H L G$ are similar triangles, the relation will be $|G L| /|B E|=|H G| /|H E|$, and it turns into Eq. (14d) for $|G L|=|D B|$ and $|H G|=|B H|$ equations.

$$
|H E|=\frac{|H G| \cdot|B E|}{|D B|}
$$


Here, Eq. (14d) is also $\left|H C^{\prime}\right|$ for $\left|H C^{\prime}\right|=|H E|$ equation. As the equation is $|A E|=\left|A C^{\prime}\right|$, then it will be $|A E|=$ $\sqrt{|A B|^{2}+\left|B C^{\prime}\right|^{2}}$, and finally turns into Eq. (14e).

$$
|B E|=\sqrt{|A B|^{2}+\left|B C^{\prime}\right|^{2}}-|A B|
$$

As we know $|H G|$ as Eq. (14c), $|D B|$ as Eq. (14b), and $|B E|$ as Eq. (14e), then also we can find out Eq. (14d) after this.

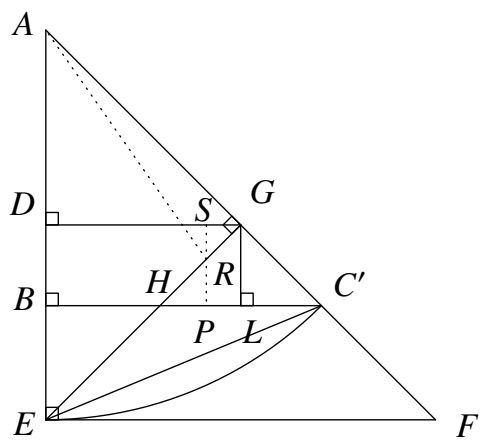

Fig. 6: A part of Fig. 5

If $A C$ radius is intersected with $E G$ by lengthening, and just a part is taken from Fig. 5, it will be as Fig. 6. As $\triangle G S R$ and $\triangle G D E$ are similar triangles, it will be Eq. (14f).

$$
\frac{|G S|}{|G D|}=\frac{|S R|}{|D E|}
$$

In $\triangle G S R$, as it will be $|G R|=\sqrt{|S R|^{2}+|G S|^{2}}$ also it will be Eq. (14g) over Eq. (14f).

$$
|G R|=\frac{|S R| \sqrt{|G D|^{2}+|D E|^{2}}}{|D E|}
$$

For $(|D B|-|S R|) /|B E|=|H R| /|E H|$ equation over the similarity between $\triangle R P H$ and $\triangle H B E,|H G|=|G R|+|H R|$ equation turns into Eq. (14h) for $|S R|$.

$$
|S R|=\frac{|D E|(|H G| \cdot|B E|-|E H| \cdot|D B|)}{|B E| \sqrt{|G D|^{2}+|D E|^{2}}-|D E| \cdot|E H|}
$$

If Eq. (14h) is used on Eq. (14f), it will be as Eq. (14i).

$$
|G S|=\frac{|G D|(|H G| \cdot|B E|-|E H| \cdot|D B|)}{|B E| \sqrt{|G D|^{2}+|D E|^{2}}-|D E| \cdot|E H|}
$$

Being the equations are as $|G R|=\sqrt{|S R|^{2}+|G S|^{2}},|E R|=$ $|E G|-|G R|,|A R|=\sqrt{|A G|^{2}+|G R|^{2}},\left|B C^{\prime}\right|=|E G|$ over the law of cosines for $\angle E A C=\alpha$, it will be as Eq. (15).

$$
\cos \alpha=\frac{|A E|^{2}+|A G|^{2}-\left|B C^{\prime}\right|^{2}+2\left|B C^{\prime}\right| \cdot|G R|}{2|A E| \sqrt{|A G|^{2}+|G R|^{2}}}
$$

\subsubsection{Area and length relations}

If an internal tangent circle is drawn in a square as the same as on Fig. 7, and if length of the square is changed forever gradually, then all the lengths of the circle in the square have to change in the same ratio; because $A D$ is radius of the circle and also $|F C|$ is related with the same radius $A F$; so also all will be emerged lengths of the circle change at the same amount that; $|E F|,|A E|,|D E|$ and $|E B|$ are included as well naturally.

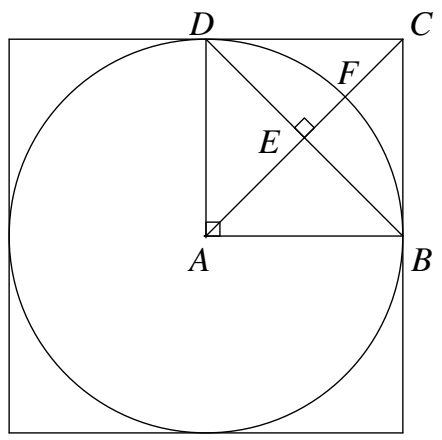

Fig. 7: Internal tangent circle

During these changing, also ratios of the area which are between $\overparen{D B}$ and $D B$ chord are always conserved, any area in $A B C D$ square is included as well; because if some triangles are drawn as depended on the rule in Fig. 8 gradually forever, then there shall always emerge some triangles with right angle instead of the circle slice; therefore the area between $\overparen{D B}$ and $D B$ chord, and $\overparen{D B}$ and $|D C|+|C B|$ always change in the same rate.

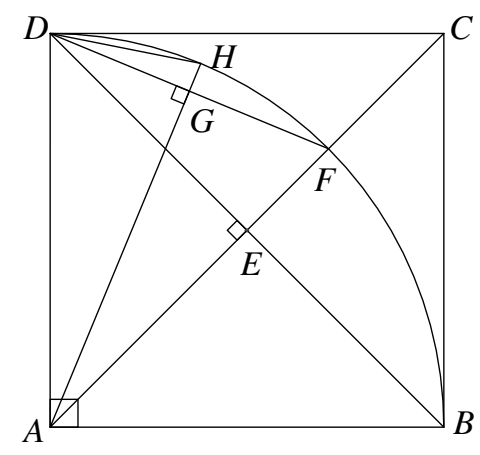

Fig. 8: The quarter slice of Fig. 7

Already it can be analyzed by looking at the radius, $\triangle A B D$ and the other chords, the arcs are included as well. It can be said that ratio of two length which are intersected or independent of each other is the same and fixed for changing radius or square sides; so also ratio of the areas which are dependent of the lengths is the same and fixed as well, where lengths and areas are amongst themselves. If this is the condition, then for 
the circle area $A, r$ radius and square area $4 r^{2}$ which emerges for the same radius, it will be a constant like $W=4 r^{2} / A$. Thereupon, let the circle's area is a number like $\pi$ where $r=1$ unit . For this radius, the equation becomes $4 r^{2} / A=4 / \pi$ over the $W$ constant. Finally for changing $r$ values, area of a circle becomes Eq. (16).

$$
A=\pi r^{2}
$$

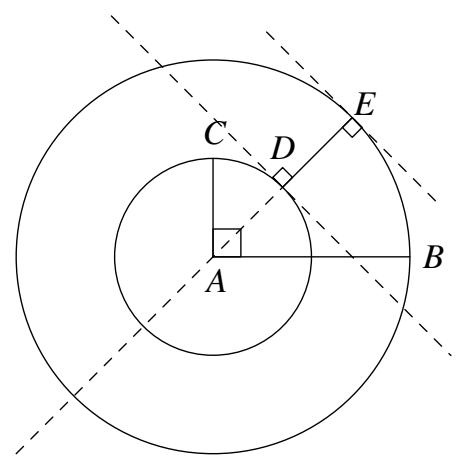

Fig. 9: The area scanned

On Fig. 9, there are two circles which were drawn nested manner, and have $|A C|=r_{1}$ and $|A B|=r_{2}$ radius. At the end of the scanning for 1 round, the area scanned by $|D E|$ becomes $A_{2}-A_{1}$ for $|A C|=r_{1}$ and $|A B|=r_{2}$ radius. During the process, the distance taken by $E$ point becomes $C_{2}$, and by $D$ point becomes $C_{1}$. For $D E$. The distance taken by infinite points between $D$ and $E$ points on $|D E|$ becomes $A_{2}-A_{1}$ as also it was derived.

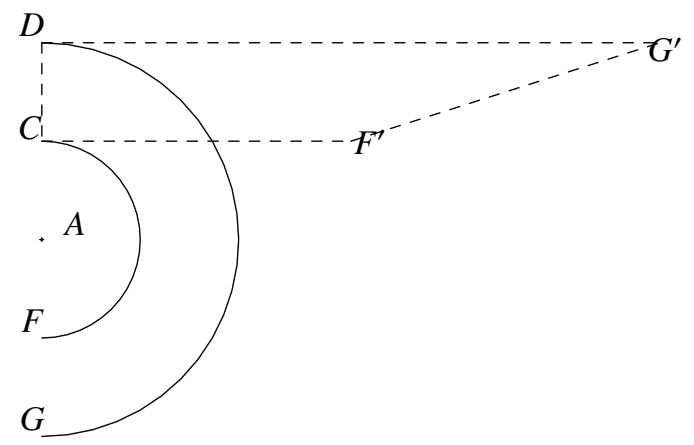

Fig. 10: The area of the circle in the kind of triangle

This process can be shown as Fig. 10 for the same radius even for half of the circle. It becomes $\left|D G^{\prime}\right|=|\overparen{D G}|=C_{2} / 2$ and $\left|C F^{\prime}\right|=|\overparen{C F}|=C_{1} / 2$. The area of the trapezoid becomes $A\left(D G^{\prime} F^{\prime} C\right)=\left(A_{2}-A_{1}\right) / 2$. While $r_{1}$ radius is approaching to 0 , the area of trapezoid approaches to $\triangle D G^{\prime} A$, and also the area is approaching to a single circle's area with $r_{2}$ radius. For the whole circle, it becomes Eq. (16a).

$$
A=\lim _{r_{1} \rightarrow 0} \pi\left(r_{2}-r_{1}\right)^{2}
$$

Hence it becomes $\pi r^{2}$ for $r_{2}=r$ as there is only 1 radius after this. For this condition, after this area of the triangle becomes $\mathrm{Cr} / 2$ where $C_{2}=C$ for the whole circle. Finally over $C r / 2=\pi r^{2}$ equation, circumference of a circle becomes Eq. (16b).

$$
C=2 \pi r
$$

\subsubsection{Computation of pi}

Relation between area and circumference of a circle is Eq. (17)

$$
C=2 A / r
$$

where $A$ is area and $C$ is circumference. If the area is an integer and the angle is an angle like $60^{\circ}$ which has a certain value for $\cos (60)$ which can be found out by using an equilateral triangle, then by using Eq. (15), $r$ can easily be found out as the circumference is $A / 3 r$ and the radius is $r$, by solving equation. By this way, also $\pi$ can be found out by infinite number of different integer area values for Eq. (18)

$$
\pi=A / r^{2}
$$

As the radius after all, if the angle, and the arc which is depended on $2 \pi r \alpha / 360$ are known on Eq. (15), then $\pi$ directly can be found out for $\cos (60)$ and $r=1$ as well by solving equation.

\section{Conclusion}

Matter is pretty process-able because of uncertainty. It always emerges by forcing and so it is absolute inert. Nothing works. As it was said [1], matter has an energy potential with a magnitude of $m v^{2}$ which has "Joule per second" that can be shorten as $(J s)$ unite for a creation velocity of $v$ because of this availability. As there is no difference for matter if it directly works or made it worked and it is static or moving, we can use matter's own energy without external energy as self-running devices forever to work of it in the name of us each second to its total energy. As we use matter's own energy, it is not so hard to convert energy into force in the other name mass. We can gain more acceleration without external energy source than Jet Fighters without heat and other negativeness. There are some examples [1], [4].

Also if I am right, we can make an identical simulation of the universe. I found out some physical values of the empty universe approximately such as its mass, volume and radius [1]; but it is not important; because the important thing is the main functions the each physical phenomena work over them. In theory, there is no difference between real life and a simulation; because both of them are an illusion as well. If we can achieve the work by quantum computers as the main computers, especially it will help astrophysics. Also we will be able to calculate interstellar jumping ship condition in one atom sensitivity. If matter draws more space while it was emerging by using the space, then it can go faster than light when the light speed is fixed at that time. Uncertainty renders this as possible. 
If we can achieve the work, also it will help us to find new worlds. We will be able to make a simulation screen that you can see the sky like there is no screen when everything is at its place on sky. We can know any planet's instant weather condition and make a carbon test for a material at the other part of the universe. If we get lost in the universe by teleportation, then we can find where are we at that time by eliminating according to geographical shapes, elements and heavenly bodies around us in theory even if you do not know any physical law, constant and subject by only thinking and using some machines. Even if we cannot find the twin of the universe by these values, we can try it each second for the astrophysical information in our hands as a reference; because even if the gravitational constant is very sensitive, the smallest difference cannot create this universe as the identical twin.

\section{References}

1. Kavak M. 2016. On the Uncertainty Principle. American Journal of Physics and Applications Vol. 4, No. 4, pp. 90-123

2. Planck M. 1901, On the Law of Distribution of Energy in the Normal Spectrum, Annalen der Physik, vol. 4, p. 553 ff.

3. Heisenberg W. 1927, The actual content of quantum theoretical kinematics and mechanics, Z. fuer Phys., Volume 43, Issue 3, pp. 172-198

4. Kavak M. 2018. Complement Inferences on Theoretical Physics and Mathematics. Open Science Framework. Available online: osf.io/tw52w 